EPJ Web of Conferences 66, 10011 (2014)

DOI: 10.1051/epjconf/20146610011

(C) Owned by the authors, published by EDP Sciences, 2014

\title{
Nuclear-physics applications of MYRRHA
}

\author{
Lucia Popescu $^{\text {1a }}$ (for the ISOL@MYRRHA and MYRRHA Collaborations) \\ ${ }^{1}$ Belgian Nuclear Research Centre SCK•CEN, Boeretang 200, B-2400 Mol, Belgium
}

\begin{abstract}
The Belgian Nuclear Research Centre SCK $\cdot \mathrm{CEN}$ is currently working on the design of the MYRRHA research reactor, able to operate in both critical and sub-critical mode as an Accelerator-Driven System (ADS). When operated as an ADS, the MYRRHA reactor core will be coupled to an external neutron source, which is generated by a 600 $\mathrm{MeV}$, 2- to 4-mA proton beam impinging on a lead-bismuth spallation target. By using a small fraction (up to 5\%) of the MYRRHA proton beam, intensities of 100-200 $\mu \mathrm{A}$ can be sent to a separate facility called ISOL@MYRRHA. Given the high proton energy, most isotopes known on the chart of nuclides can be produced. The production in the hot-target is followed by selective ionization and extraction of atoms in a Radioactive Ion Beam (RIB). Following mass-purification, high-intensity RIBs will be delivered for a large variety of experimental programmes requiring long measurement times. By its experimental programme, the ISOL@MYRRHA facility will be complementary to running and planned Isotope Separator On-Line (ISOL) facilities in Europe and abroad.
\end{abstract}

\section{Introduction}

MYRRHA, (Multi-purpose hYbrid Research Reactor for High-tech Applications), is a hybrid system, that combines a $600-\mathrm{MeV}$ x $4-\mathrm{mA}$ proton linear accelerator and a lead-bismuth eutectic (LBE) cooled fast spectrum research reactor. [1,2] MYRRHA can be operated in both sub-critical (acceleratordriven system (ADS)) and critical modes, allowing fuel developments for innovative reactor systems, material developments for GEN IV systems, material developments for fusion reactors, medical radioisotopes production and industrial applications (e.g. Si-doping). The system will also demonstrate the ADS full concept by coupling the three components (accelerator, spallation target and subcritical reactor) at a reasonable power level to allow operation feedback, scalable to an industrial demonstrator, and allow the study of efficient transmutation of high-level nuclear waste.

Moreover, by branching off a small fraction of the proton beam, the ISOL@MYRRHA facility will be operated in parallel to the MYRRHA-ADS. [3] This facility should use up to 200- $\mu$ A x 600$\mathrm{MeV}$ proton beam for the production of Radioactive Ion Beams (RIBs) via the Isotope Separator On Line (ISOL) method. By combining the high primary-beam intensity with selective ionization and a beam-purification system with high mass-resolving power, it will be possible to produce intense RIBs with high purity. The ISOL@MYRRHA facility aims to be complementary to existing facilities, by focusing on experimental programs in need of long uninterrupted beam times. These are experiments which:

\footnotetext{
${ }^{\mathrm{a}}$ Corresponding author: lpopescu@sckcen.be
}

This is an Open Access article distributed under the terms of the Creative Commons Attribution License 2.0, which permits unrestricted use, distribution, and reproduction in any medium, provided the original work is properly cited. 
- $\quad$ hunt for very rear phenomena,

- need high statistics,

- need many time-consuming systematic measurements,

- have inherent limited detection efficiency.

Figure 2. The concept of MYRRHA with the ISOL extension.

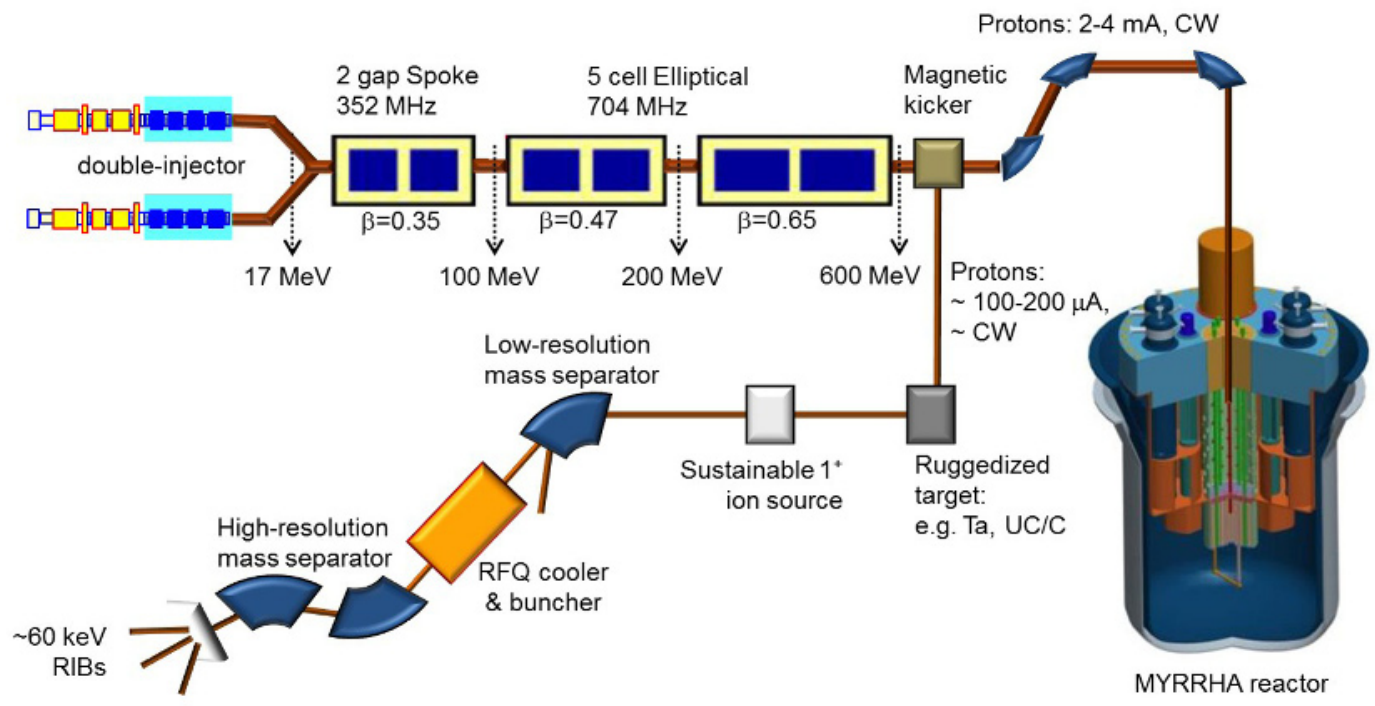

\section{Applications of MYRRHA}

2.1 Sustainable fission energy: demonstrate the physics and technology of an Accelerator Driven System (ADS) for transmuting long-lived radioactive waste

The minor actinides are the part of the high-level nuclear waste which combines a high radiotoxicity and a long lifetime. Their elimination by transmutation would not exempt the waste from underground disposal, but it would substantially reduce the size of these deposits and the required duration of the disposal. The MYRRHA project will allow the demonstration and performance assessment of the transmutation concept. This facility is a first research tool for the demonstration of the process's efficiency. Through MYRRHA, we establish the procedures to be used during transmutation exercises and obtain useful information for the next generation industrial transmutators. [2]

\subsection{Sustainable energy: development of a fast-spectrum reactor and fusion technology}

Sustainable nuclear energy development requires the gradual transition of the current thermalspectrum reactors to fast-spectrum reactors. For this, innovative fuels and materials need to be tested and qualified for the future GEN IV fast-reactor concepts. Also for the development of fusion energy the challenges lay to a large extent in the area of materials. The development of materials and fuels can only be performed in an irradiation facility where fully controlled and representative experimental conditions can be obtained. Thanks to its fast spectrum and spallation target, MYRRHA will be a flexible irradiation facility that can fulfil this need. 
Although MYRRHA is especially suited for testing and demonstrating the proper working of different technological components for the heavy liquid metal based reactor concepts (e.g. Lead Fast Reactor), MYRRHA will aim at testing new technological developments for sensors and instrumentation for innovative fission reactors, fusion reactors and space applications.[2]

\subsection{Enabling technologies for renewable energies: production of neutron irradiated silicon}

Renewable energies (such as windmills, solar panels and hybrid/electric cars) rely on power electronics. These power electronics are essentially based on semiconductors that are fabricated from neutron-irradiated silicon in order to obtain the required homogeneous resistivity. This is currently being performed in materials testing reactors (MTR's) such as BR2 and is planned to be continued in MYRRHA.[2]

2.4 Health care: production of radioisotopes for nuclear medicine

Radioactive solutions for cancer diagnosis are prepared and dispatched every day to hospitals around the world. Up to $80 \%$ of these contain an isotope of technitium $\left({ }^{99} \mathrm{Tc}\right)$ obtained from the decay of molybdenum $\left({ }^{99} \mathrm{Mo}\right)$ produced in Europe by the irradiation of uranium targets in three reactors including BR2. To guarantee the continuity of the production of radioisotopes with a short lifetime, the availability of at least one reactor is required at any time. The intermittent way of operation of such reactors justifies the need for at least three supply sources in Europe. Moreover, since these reactors are reaching the end of their foreseen lifetime, it is considered to replace them by new systems. MYRRHA will allow $\mathrm{SCK} \cdot \mathrm{CEN}$ to pursue the supply of medical isotopes after the decommissioning of BR2.[2]

2.5 Science: production of radioisotopes for fundamental and applied science via the Isotope Separation On-Line method at ISOL@MYRRHA [3]

The envisaged scenario is a parallel operation of MYRRHA and ISOL@MYRRHA. The 200- $\mu$ s "holes" in the proton-beam requested by the MYRRHA reactor are taken out to the ISOL@MYRRHA facility at a repetition rate which can range from 125 to $250 \mathrm{~Hz}$ for a corresponding $100-$ to $200-\mu \mathrm{A}$ average beam current. This will be realized by implementing a magnetic kicker followed by a magnetic septum once the two beams are separated.

ISOL@MYRRHA will follow closely the RIB production schemes that are developed and successfully used at the ISOLDE-CERN and ISAC-TRIUMF facilities. It will be equipped with ruggedized target-ion source systems that allow the use of a selection of target materials, including actinide targets, which can withstand the proton-beam power (up to $\sim 100 \mathrm{~kW}$ ) for extended time periods without compromising the yield of particular radioactive isotopes. Three types of ion sources are foreseen for selective ionization of the products: the hot-surface ion source, the resonant ionization laser ion source (RILIS) and the electron cyclotron resonance ion source (ECRIS). Additional purification occurs by mass separation after extracting the ions over a potential difference of up to 60 $\mathrm{kV}$ forming the radioactive ion beam.

In order to make effective use of the beam time, the parallel multi-users aspect of ISOL@MYRRHA is an important aspect in the design study. Since a high-resolution mass separator prevents the use of different beams at the same time, a pre-separator with low mass resolution is considered. In this way, one could envisage a scenario in which lower-mass isotopes (like, e.g., ${ }^{8} \mathrm{Li}$ for $\beta$-NMR studies) are used for solid-state physics in parallel with an experiment using heavier nuclei. Moreover, the pre-separation avoids a too intense RIB loading of the RFQ cooler and buncher, which allows a high-quality low-emittance beam. As a result, the high-resolution magnet can be maximally exploited with a mass-resolving power $\mathrm{M} / \Delta \mathrm{M}$ in the order of $10^{4}$.

Measurements with high-intensity beams and long/regular beam times are an important source of information for quasi all fields in science making use of RIBs, ranging from fundamental interaction 
measurements with extremely high precision over systematic measurements for condensed-matter physics and production of radio-isotopes. Experiments, which need very high statistics, need many time-consuming systematic measurements, hunt for very rare events, and/or have inherent limited detection efficiency are particularly interested in extended beam times. Long beam times could be of interest for astrophysics, when nuclear reactions with small cross sections are involved, but the absence of a post-accelerator in the present phase of ISOL@MYRRHA will prevent such kind of studies. Although higher-energy secondary beams are not discarded for a later phase, for the moment only physics cases with beam energies up to $60 \mathrm{keV}$ are being considered. [4]

\subsubsection{Applications in Atomic Physics}

By applying atomic-physics techniques like mass, laser, and radiofrequency spectroscopy, key information can be obtained on properties of nuclear ground and isomeric states. These are atomic masses, the nuclear spin I, the magnetic moment $\mu_{\mathrm{I}}$, the hyperfine anomaly ${ }_{1} \Delta_{2}$ between two isotopes (indicated by the subscripts 1 and 2) of an element, the spectroscopic nuclear quadrupole moment $\mathrm{Q}_{\mathrm{s}}$, and changes in the mean-square nuclear charge radius $\delta<\mathrm{r}^{2}>^{\mathrm{A}, \mathrm{A}^{\prime}}$ between isotopes with mass numbers $\mathrm{A}$ and $\mathrm{A}^{\prime}$. But also the opposite relation between atomic and nuclear physics holds true, as quantum electrodynamics (QED) can be tested in the strong-field limit of highly-charged ions.

High sensitivity and/or precision is needed for, e.g., studying weakly-produced beams or observing weak effects. Such studies can only be accomplished if long beam time is available for performing extended systematic studies and for acquiring the necessary statistics. If sufficient precision is reached, hyperfine anomalies can be investigated by combining laser and radiofrequency techniques. Systematic data from several isotopes of one element can be compared to nuclear models. Moreover, there is an increased interest for hyperfine anomaly since theoretical work has shown the possibility to connect the hyperfine anomaly to the change in neutron radius $\left.\delta<\mathrm{r}_{\mathrm{N}}^{2}\right\rangle$, as compared to the change in the charge radius $\delta<\mathrm{r}_{\mathrm{C}}{ }^{2}>$. Except for a few cases, no systematic measurements exist yet.

Highly-charged heavy ions provide a unique testing ground for quantum electrodynamics (QED) in very strong electric and magnetic fields, which is experimentally not accessible otherwise. The electric-field strength experienced by the 1 s-electron in, e.g., $\mathrm{H}$-like $\mathrm{U}^{91+}$ is about $10^{6}$ times larger compared to $\mathrm{H}^{+}$. Once QED corrections are under control, the hyperfine fields of $\mathrm{H}$-like ions can be safely treated by the atomic theory. Consequently, one can determine with very high accuracy absolute nuclear charge radii, nuclear magnetic moments and the Bohr-Weisskopf effect in the isotope series of heavy elements.

Polarized radioactive beams are easily achieved using, e.g., resonant collinear laser excitation with circularly polarized laser light [5]. The availability of polarized radioactive beams will be crucial for several research projects. Examples are $\beta$-NMR in condensed-matter research $\left({ }^{8} \mathrm{Li}\right)$ and biosciences (elements like $\mathrm{Cu}$ ), and correlation measurements for weak-interaction studies.

\subsubsection{Applications in Nuclear Physics}

In nuclear physics, determining precise values of extremely small decay branches (in the order of $10^{-6}$ ) or crystal $\gamma$-ray spectrometry with very high resolution can provide crucial experimental input for understanding aspects of nuclear structure. The focus will be on $\beta$-decay and $\beta$-delayed particle-decay studies over other decays. In particular, systematic measurements of $\beta$-delayed neutron probabilities $\mathrm{P}_{\mathrm{n}}$ are of special interest in nuclear astro-physics to model the nuclear paths of the rapid-neutron process, as well as in nuclear applications as nuclear-reactor control and non-destructive characterization of nuclear waste. The required equipment will consist of a passive or active stopper, or a trap catching the radioactive ion to be investigated, and detectors for $\beta, \gamma$, charged particles and neutrons. In order to obtain the desired statistics with such rare-event decays or inherent limited detection efficiencies of crystal spectrometers, not only high-intensity beams, but also the availability of long beam times can be of crucial importance. 
The Gamow-Teller strength B(GT) distribution in a nucleus provides an important test of structure calculations for that nucleus. A major part of the $\mathrm{B}(\mathrm{GT})$ strength, however, is situated in the GamowTeller Giant Resonance (GTGR). Although $\beta$ transitions to the GTGR have a large B(GT) strength, the corresponding $\beta$-branching ratios are small as a consequence of the small phase-space factor $f$.

Strength distributions can be directly measured with particle detectors. However, it can also be indirectly determined by measuring the line shape of gamma transitions after $\beta$-delayed particle decay. Moreover, additional information can be deduced like the half-life of the excited state from which the $\gamma$ transition arises and other resonance parameters. A recent example is the $\beta$-delayed neutron decay study of ${ }^{11} \mathrm{Li}$ measured with the $8 \pi$ spectrometer, an array of 20 Compton-suppressed HPGe detectors, see also picture. [6] A much more precise determination of the line shape can be obtained, however, by using a $\gamma$-ray crystal spectrometer, which allows $\gamma$-ray energy resolutions down to the ppm precision in the energy domain 0.1-6 MeV. Its inherent low detection efficiency has to be compensated by the intense ISOL@MYRRHA RIBs and long beam times.

When multi-particle decays occur (e.g., $\beta \mathrm{xn}, \beta \mathrm{xp}, \beta \mathrm{pn}$ ), one of the key questions concerns the particle-emission mechanism that is involved. This can be investigated by measuring the correlation between the decaying particles. Although the $\beta$-delayed multi-particle process is energetically allowed in many nuclei and should occur in many more than the presently known emitters [7], information on the particle-emission mechanisms is very scarce.

Another important topic concerns the halo nuclei. The three most studied halo nuclei are ${ }^{6} \mathrm{He},{ }^{11} \mathrm{Li}$, and ${ }^{11} \mathrm{Be}$. However, a few others, such as ${ }^{14} \mathrm{Be},{ }^{14} \mathrm{~B},{ }^{15} \mathrm{C}$ and ${ }^{19} \mathrm{C}$ are waiting for a more thorough experimental and theoretical study. These systems can typically decay through different $\beta$-delayed (multi-) particle channels at high excitation energies in the daughter and, thus, (extremely) low branching ratios are involved. If high-precision data can be obtained, these decays provide, however, a detailed test of halo wave functions, for which microscopic ab-initio calculations with realistic nucleon-nucleon and even nucleon-nucleon-nucleon interactions can be performed.

\subsubsection{Applications in Fundamental Interactions Physics}

Weak-interaction studies and symmetry tests in neutral atoms and ions are used for investigating fundamental interactions and testing the standard model via, e.g., measuring Ft values and investigating specific correlations in the $\beta$-decay process as precise as possible. As these experiments strive to the highest possible precision, the systematic errors in the experimental setup have to be understood as good as possible and large statistics are of primordial importance.

By simultaneously measuring physical observables such as energy, momentum and spin of the particles involved in nuclear $\beta$ decay, either by direct or indirect methods, correlation coefficients between those observables can be determined. Because the correlation coefficients depend on the coupling strengths for the different possible weak-interaction types, being vector (V), axial vector (A), scalar (S) and tensor (T) coupling, high-precision measurements allow to test the standard model, which assumes only $\mathrm{V}$ - and A-type interactions and with maximal parity violation and time reversal invariance. The use of open trap structures, such as Penning, Paul, and MOT traps is an absolute requirement in order to avoid significant systematic uncertainties.

It is well known that the Conserved Vector Current (CVC) hypothesis and the unitarity of the $\mathrm{CKM}$ matrix can be tested by using Ft values of super-allowed $0^{+} \rightarrow 0^{+}$Fermi transitions. Less well known, however, is that super-allowed decays of $\mathrm{T}=1 / 2$ mirror transitions offer a potentially more sensitive probe when its Ft value is measured in combination with the ratio $\varphi$ of the Gamow-Teller to Fermi amplitudes, which can be determined by correlation measurements. So far, only few cases have been studied and there is much room for improvement. [4]

By performing high-precision measurements on atoms with high atomic number $\mathrm{Z}$, symmetries can be tested in the electroweak interaction. High- $Z$ atoms are more sensitive to possible new shortranged interactions between leptons and quarks, because the overlap of electrons with the nucleus is larger. Beams from ISOL@MYRRHA would allow exploring these possibilities using state-of-the-art atomic experimental techniques. An obvious program would be the study of time reversal violation by 
measuring the electric dipole moment (EDM) of atoms like radium. Another possibility would be atomic parity non-conservation (APNC) experiments with francium atoms.

\subsubsection{Applications in Condensed Matter and Biology}

In condensed-matter physics and biology, techniques like ${ }^{8} \mathrm{Li} \beta$-NMR and emission channelling could profit from the available long beam times or from the frequent access to short beam times. Systematic measurements allow investigating a larger range of samples with varying parameters, which give rise to a more detailed study.

The progress in applications (especially in the electronics industry) is firmly rooted in fundamental studies of nanostructured materials, where mesoscopic effects become very pronounced due to the fact that the system size is comparable to relevant physical length scales. One of the key features in understanding the physics of nanostructured materials is to have microscopic information about the local electric and magnetic field within the sample. Very often these fields may vary significantly as function of, for instance, the depth within the sample. In $\beta$-NMR, a polarized radioactive beam (e.g., ${ }^{8} \mathrm{Li}$ ) is implanted in the sample. The implantation depth is controlled by varying the electrostatic potential on the sample, which defines the beam energy. The spin rotation of the implanted radioactive isotope depends on the local hyperfine field. By measuring the $\beta$ asymmetry and its disappearance at the RF resonances, one can therefore extract information on the local magnetic and electric fields. Since several years, $\beta$-NMR is used very successfully for condensed-matter studies at the TRIUMF facility in Vancouver. [8]

The presence of impurity atoms can drastically change the electrical, magnetic and optical properties of semiconductors. To understand the effect of impurities on the properties of the material, it is important to know the exact lattice location (in other words the local crystal field) of these impurities, and how the lattice site depends on the presence of defects in the semiconductor. Direct information on the lattice site of impurity atoms can be provided by approaches relying on the channelling of charged particles. In emission-channelling experiments $[9,10]$, radioactive probe atoms - acting as emitters of (conversion) electrons, positrons, or $\alpha$ particles - are implanted in the single crystalline host. During the subsequent decay, the emitted charged particles can channel along a major symmetry direction (lattice axis or plane) or can be blocked, resulting in anisotropic emission patterns. The yield of charged particles emitted from the radioactive impurity atoms inside the sample is recorded with a two-dimensional position sensitive detector for different emission directions around major crystallographic axes and planes, where channelling or blocking effects are experienced. This technique gives rise to establishing with high sensitivity the lattice-site positions of impurity atoms with a precision below $0.1 \AA$.

\subsubsection{Applications in Nuclear Medicine}

The wide variety of medical radioisotopes for both therapy and imaging are directly accessible at an ISOL facility. Most importantly, an ISOL facility provides a universal means to produce essentially all isotopes of medical interest practically carrier free and of high purity. Consequently, one can enable systematic biokinetic studies, simultaneously with different isotopes and different tracers. Besides the research for new and innovative medical radioisotopes, such isotopes can be systematically produced in very clean conditions with the extended beam times available at ISOL@MYRRHA.

Single cancer cells and small cancer cell clusters can be effectively targeted with a tumour-seeking tracer labelled with $\alpha$-emitting radioisotopes. The tumour-seeking tracer is a molecule that specifically binds to the cancer cells and the $\alpha$-emitting radioisotopes attached to the tracer deliver the required dose. Alpha-emitting radioisotopes may be of great advantage in this kind of therapy, because of the shorter penetration track compared to $\beta$ and $\gamma$ radiation. It has been shown that only very few $\alpha$ hits are sufficient to kill a cell, while the short range of the $\alpha$ particles $(30-80 \mu \mathrm{m})$ increases the safety profile of $\alpha$ emitters. Nonetheless, the $\alpha$-emitting nuclide has to fulfil several important requirements 
such as half-life, radiotoxicity of the daughter isotopes, biokinetics (in-vivo stability of the tumourseeking tracer, clearance,...), affordable production price, and reliable supply.

It has been demonstrated that single cancer cells can be sterilized with high efficiency using rituximab labelled with ${ }^{149} \mathrm{~Tb}$ [11]. The study was carried out on 26 SCID (Severe Combined Immuno-Deficient) mice, which, being deficient in the immune defence of so-called B- and T-cancer cells, easily develop tumour masses after injection of such cancer cells. As shown by Beyer et al. [11], only the mice treated with the ${ }^{149} \mathrm{~Tb}$-labelled rituximab were almost completely protected and did not show any signs of disease for four months after injection.

\section{Acknowledgements}

Contributions are acknowledged from: H. Aït Abderrahim, P. Baeten, D. Pauwels, P. Schuurmans, M. Schyns, J. Wagemans (SCK•CEN, Belgian Nuclear Research Centre, B-2400 Mol, Belgium), and M. Huyse, G. Neyens, R. Raabe, N. Severijns, K. Temst, P. Van Duppen, A. Vantomme (Instituut voor Kern- en Stralingsfysica, KU Leuven, B-3001 Leuven, Belgium).

The physics case of ISOL@MYRRHA is based on ideas presented during the 'Nuclear Physics Research at the MYRRHA Accelerator' BriX workshop 2008 in Mol (Belgium) [12] by: H. A. Abderrahim, B. Blank, Y. Blumenfeld, M. Dombsky, Y. Kadi, H.-J. Kluge, U. Köster, J. Lettry, A. Mueller, V. Ricciardi, D. Ridikas, K. Riisager, Z. Salman, D. Schumann, N. Severijns, U. Wahl and H. Wilschut.

\section{References}

1. 'MYRRHA, a Multipurpose hYbrid Research Reactor for High-end Applications' H. A. Abderrahim, P. Baeten, D. De Bruyn, J. Heyse, P. Schuurmans, J. Wagemans, Nuclear Physics News, 20: 1, $24-28$ (2010)

2. http://myrrha.sckcen.be

3. http://isolmyrrha.sckcen.be

4. ISOL@MYRRHA report: ISOL@MYRRHA - An On-Line Isotope Separator coupled to the MYRHHA Proton Accelerator', edited by D. Pauwels and P. Schuurmans, http://isolmyrrha.sckcen.be/ /media/Files/ISOL/Documents/ISOLMYRRHAreport.pdf

5. C.D.P. Levy et al., Nucl. Instr. Meth. B, 204, 689 (2003)

6. F. Sarazin et al., Phys. Rev. C, 70, 031302(R) (2004)

7. B.Jonson ans K. Riisager, Nucl. Phys. A, 693, 77 (2001)

8. G.D. Morris et al., Phys. Rev. Lett., 93, 157601 (2004)

9. H. Hofsäss and G. Lindner, Phys. Rep., 201, 121 (1991)

10. U. Wahl et al., Nucl. Instr. and Meth. A, 524, 245 (2004)

11. G.-J. Beyer et al., Eur. J. Nucl. Med. Mol. Imaging, 31, 547 (2004)

12. http://iks32.fys.kuleuven.be/wiki/brix/index.php/Workshops\#.22Nuclear_Physics_Research_at th e MYRRHA_Accelerator.22 Workshop 
